\title{
Caracterización y seguimiento durante seis meses de una cohorte de recién nacidos con sífilis congénita
}

\author{
Cristian Vallejo ${ }^{1}$, Yolanda Cifuentes ${ }^{2}$ \\ 1 Departamento de Pediatría, Facultad de Medicina, Universidad Nacional de Colombia, Bogotá, D.C., Colombia \\ 2 División de Neonatología, Departamento de Pediatría, Facultad de Medicina, Universidad Nacional de \\ Colombia, Bogotá, D.C., Colombia \\ Lugar donde se desarrolló la investigación: Hospital La Victoria, sede Instituto Materno Infantil, Bogotá, \\ D.C., Colombia
}

Introducción. La sífilis congénita es una enfermedad prevenible cuya incidencia en Colombia ha aumentado, alcanzando la cifra de 3,28 casos por 1.000 nacidos vivos en el 2011.

Objetivo. Caracterizar a los recién nacidos con sífilis congénita y describir su diagnóstico y tratamiento durante el seguimiento de seis meses.

Materiales y métodos. En el periodo comprendido entre agosto de 2011 y febrero de 2012 en el Hospital La Victoria, sede Instituto Materno Infantil, se identificaron y trataron los recién nacidos con sífilis congénita, y se hizo seguimiento clínico y de laboratorio hasta la curación de la enfermedad. Las variables analizadas incluyeron las características clínicas y de laboratorio.

Resultados. En este periodo se diagnosticaron 29 pacientes que cumplían con los criterios de caso de sífilis congénita, cuya frecuencia en la institución era de un caso por cada 69 nacimientos. Hubo un mortinato y una muerte neonatal, cuatro recién nacidos fueron prematuros y cinco presentaron restricción en el crecimiento. De los 28 recién nacidos vivos, 15 fueron asintomáticos. Se presentaron dos casos con neurosífilis, es decir, $15 \%$ de los sintomáticos. Se hizo seguimiento a 18 de los 27 niños (66,6\%), incluidos la mayoría de los sintomáticos y los dos casos con neurosífilis; la disminución esperada de los títulos en la prueba del Venereal Disease Research Laboratory, VDRL, se presentó en todos los pacientes, y no hubo secuelas clínicas, con lo que se cumplieron los criterios de curación.

Conclusiones. Se evidenció una gran incidencia de la enfermedad, fallas en su prevención, una variada expresión en su presentación y la efectividad del tratamiento en el periodo neonatal.

Palabras clave: sífilis congénita, neurosífilis, retardo del crecimiento fetal, mortalidad infantil, mortinato, estudios de seguimiento.

doi: http://dx.doi.org/10.7705/biomedica.v36i1.2661

\section{Characterization and six-month follow-up on a cohort of newborns with congenital syphilis}

Introduction: Congenital syphilis is a preventable disease. However, the incidence in Colombia has increased and reached the figure of 3.28 cases per 1,000 live births in 2011.

Objective: To characterize newborns with congenital syphilis and describe follow-up over 6 months following their diagnosis and treatment.

Materials and methods: Between August, 2011, and February, 2012, in Hospital La Victoria, sede Instituto Materno Infantil, newborns with congenital syphilis were identified and treated. Clinical and laboratory follow-up was carried out for six months until it was determined that the disease was cured. The analyzed variables included patients' clinical and laboratory characteristics.

Results: In this period, we identified 29 cases that met the criteria of congenital syphilis, with a frequency in the institution of one case per 69 births. There was one stillbirth and one neonatal death, four were premature, and five had growth restriction. Of the 28 live births, 15 were asymptomatic. There were two cases with neurosyphilis, representing $15 \%$ of symptomatic newborns. Follow-up was done on 18

\footnotetext{
Contribución de los autores:

Cristian Vallejo: elaboración del consentimiento informado y presentación ante el Comité de Ética de la Universidad Nacional; búsqueda de los casos en el hospital; recolección de la información; revisión de historias clínicas; primer contacto con las madres para consecución del consentimiento informado; examen físico de los recién nacidos y, posteriormente, de los lactantes en los controles; tabulación de los datos; búsqueda y contacto con las madres para el control.

Yolanda Cifuentes: generación de la propuesta de trabajo; trámite para la financiación del trabajo; análisis y seguimiento de los casos complejos; tabulación y análisis de los datos; asistencia en el control de los pacientes complejos. Los dos autores participaron en la escritura del manuscrito.
} 
out of 27 children (66.6\%), including most symptomatic cases and the two cases of neurosyphilis. All cases showed the expected decrease in VDRL titers without clinical sequelae, meeting the criteria of being cured.

Conclusions: A high incidence of the disease, failure to prevent it, varied expression in its presentation, and the effectiveness of treatment in the neonatal period were evidenced in this study.

Key words: Syphilis, congenital; neurosyphilis; fetal growth retardation; infant mortality; stillbirth; follow-up studies.

doi: http://dx.doi.org/10.7705/biomedica.v36i1.2661

La sífilis congénita es una enfermedad prevenible que representa un grave problema de salud pública en los países en desarrollo. Los índices de mortalidad, morbilidad y discapacidad infantil a corto y largo plazo, siguen siendo elevados, a pesar de la implementación de los programas de prevención, diagnóstico y tratamiento (1). En el 2008, se estimó que la sífilis de la mujer gestante a nivel mundial había originado 305.000 muertes fetales y neonatales y que 215.000 recién nacidos tuvieron peso bajo, prematuridad o complicaciones relacionadas con la enfermedad (2). Latinoamérica y el Caribe poseen la tasa más alta de sífilis en el mundo y se cree que cada año nacen 164.000 niños con la enfermedad (3). En el 2011 se estimó una incidencia de 3,28 casos por 1.000 nacidos vivos en Colombia, cifra que varió según la región y la calidad de los sistemas de notificación obligatoria, y estuvo muy lejana de la propuesta por la Organización Panamericana de la Salud de 0,5 casos por 1.000 recién nacidos $(3,4)$.

La presencia de sífilis congénita se ha asociado a factores de riesgo materno, entre los que se destacan las prácticas sexuales riesgosas, el inicio temprano de actividad sexual, la promiscuidad, el consumo de alcohol o sustancias psicoactivas, la residencia en zonas de alta incidencia, el bajo nivel socioeconómico, la dificultad en el acceso a los servicios de salud, la ausencia o el inadecuado control prenatal, y la presencia de otras infecciones de transmisión sexual (5).

La sífilis congénita se presenta cuando la mujer con sífilis transmite la infección al feto durante la gestación por vía hematógena a través de la placenta, o durante el parto por contacto directo con lesiones infecciosas. La probabilidad de infección vertical varía según el estadio de la infección en

Correspondencia:

Cristian Vallejo, Transversal 16 bis № 45D-63, apartamento, 506 , Bogotá, D.C., Colombia

Teléfono: (316) 734 9033; fax: (571) 2338979

cristian_gv@hotmail.com

Recibido: 26/12/14; aceptado: 19/08/15 la mujer gestante no tratada, siendo de 60 y hasta $100 \%$ en los casos de sífilis primaria, de $67 \%$ en los de sífilis secundaria, de $40 \%$ en los de sífilis latente temprana y de $10 \%$ en los de sífilis latente tardía $(5,6)$.

El cuadro clínico en el recién nacido incluye desde casos asintomáticos hasta formas graves de la enfermedad, incluso hidropesía fetal; las manifestaciones tempranas se denominan así si aparecen en los primeros dos años de vida, y tardías, si aparecen después. Entre las manifestaciones tempranas puede darse el compromiso de diversos órganos y sistemas: el hígado, el bazo, el pulmón, la piel, el riñón, los ojos, el sistema hematológico y el sistema nervioso central $(5,7,8)$; sin embargo, en la mayoría de los casos, los recién nacidos con sífilis congénita no presentan manifestaciones clínicas de la enfermedad (7).

La neurosífilis se presenta en aproximadamente $80 \%$ de los neonatos con sífilis congénita diagnosticada clínicamente o confirmada por laboratorio, y en $8 \%$ de los recién nacidos sin manifestaciones clínicas ni confirmación por laboratorio (9). Los hallazgos en el líquido cefalorraquídeo sugestivos de neurosífilis son la pleocitosis, con más de 25 leucocitos por $\mathrm{mm}^{3}$, la linfocitosis o la monocitosis, y la elevación de las proteínas a más de 150 mg/ dl en el recién nacido a término y de $170 \mathrm{mg} / \mathrm{dl}$ en los prematuros. Una prueba de Venereal Disease Research Laboratory (VDRL) reactiva en líquido cefalorraquídeo, es indicativa de neurosífilis; la infección del sistema nervioso central también puede presentarse sin que se registren cambios en el análisis citoquímico $(7,9,10)$.

La confirmación diagnóstica se hace por el aislamiento de Treponema pallidum en la placenta, el cordón umbilical o en material de necropsia, o mediante la detección del microorganismo mediante reacción en cadena de la polimerasa (PCR), o con base en los resultados positivos en las pruebas serológicas de IgM específica para $T$. pallidum (Fluorescent Treponemal Antibody Absorption, FTA-ABS; Enzyme-Linked ImmunoSorbent Assay, 
ELISA, o Western Blot, WB). Frecuentemente, la determinación de IgM específica es negativa (11) y esto hace muy difícil confirmar el diagnóstico, por lo que la definición de caso de sífilis congénita se basa en el diagnóstico de sífilis en la madre gestante, sumado a la ausencia de tratamiento o a la administración de uno inadecuado (12).

El tratamiento de elección de la sífilis congénita es la administración de penicilina cristalina durante 10 a 14 días por vía intravenosa, en dosis de 50.000 unidades por kilogramo de peso cada 12 horas durante la primera semana de vida y, a partir de la segunda semana y durante el primer mes, cada 8 horas; no se ha demostrado resistencia de $T$. pallidum a la penicilina $(7,13)$.

El seguimiento de los recién nacidos se hace mediante revisión clínica y con estudios serológicos hasta que las pruebas dejen de ser reactivas; la disminución de los títulos de la VDRL debe ser de dos diluciones en tres meses, aunque es más lenta si el tratamiento se inicia después del primer mes de vida. En caso de neurosífilis, debe hacerse punción lumbar a los seis meses; para considerar que ha habido curación, la VDRL no debe resultar reactiva y los valores de proteínas y células deben ser normales (8).

Se ha observado que el seguimiento de estos pacientes no se realiza según la norma, por lo cual este trabajo pretendió caracterizar una cohorte de recién nacidos que cumplían con los criterios de caso de sífilis congénita y recibieron tratamiento en el Hospital La Victoria, sede Instituto Materno Infantil, con el fin de determinar a lo largo de seis meses de seguimiento el comportamiento clínico y los resultados en la prueba de VDRL.

\section{Materiales y métodos}

Se hizo una investigación de tipo descriptivo prospectivo en la que se incluyeron todos los recién nacidos cuyas madres hubieran tenido una prueba no treponémica reactiva (VDRL) y una prueba treponémica positiva (FTA-ABS), y no hubieran recibido tratamiento o se les hubiera administrado uno inadecuado.

Se utilizaron las siguientes definiciones (11): el caso probable sería el de un recién nacido cuya madre hubiera tenido sífilis detectada en el momento del nacimiento y no hubiera sido tratada, o se hubiera sometido a un tratamiento inadecuado, sin tener en cuenta los signos de sífilis en el recién nacido, o un recién nacido con resultado reactivo en la prueba treponémica y, además, evidencia de sífilis congénita en el examen físico, en la radiografía de huesos largos, o resultado reactivo de la VDRL en líquido cefalorraquídeo, elevación del conteo celular o de proteínas en el líquido cefalorraquídeo (sin otra causa), o resultados positivos para IgM en la FTA ABS19S o en la ELISA.

El tratamiento materno inadecuado (modificado) se definió como la administración de un antibiótico diferente a la penicilina a la mujer gestante, cuando el intervalo entre la administración del esquema de tratamiento con penicilina benzatínica y el parto fuera menor de 30 días, o cuando no hubiera habido la disminución esperada de títulos en la VDRL en el momento del parto comparado con los resultados en el momento del diagnóstico.

La disminución de los títulos en la sífilis primaria debe ser de dos diluciones entre los tres y cuatro meses, y cuando se trata de sífilis secundaria, de dos diluciones en seis meses (14). Se excluyeron los hijos de mujeres gestantes con prueba no treponémica reactiva menor de 1:8 diluciones y sin prueba treponémica confirmatoria, así como los recién nacidos remitidos a otra institución.

Semanalmente se detectaron los recién nacidos que cumplían con la definición de caso reportados en el Comité de Infecciones del Hospital, y se verificó que las madres tuvieran prueba treponémica positiva. Después de obtener el consentimiento informado, se diligenció un formato donde se consignaron los datos de la historia clínica. A los recién nacidos se les practicó el examen físico, se les tomó una radiografía de huesos largos y se les hizo valoración por oftalmología, además de los siguientes análisis: prueba de VDRL en suero, hemograma, cuantificación de bilirrubinas diferenciadas y aminotransferasas, uroanálisis, punción lumbar a las 72 horas de vida para la VDRL y examen citoquímico en líquido cefalorraquídeo.

Todos los recién nacidos recibieron tratamiento con penicilina cristalina en la dosis estándar durante 10 días; a los neonatos con neurosífilis se les administró penicilina cristalina durante 14 días.

A los tres meses de edad, se les hizo un examen físico y se tomaron muestras para la prueba de VDRL en suero, así como control de los resultados de laboratorio que habían presentado alteraciones en el momento del nacimiento. En los casos de neurosífilis, el seguimiento serológico y en líquido cefalorraquídeo se hizo a los tres meses de edad en uno de los casos y, en el otro, a los siete meses. El seguimiento terminó cuando los pacientes cumplieron con los criterios de curación. 


\section{Análisis de resultados}

Todas las variables cualitativas se analizaron mediante el cálculo de frecuencias absolutas y relativas, y las cuantitativas, con el cálculo de frecuencias y medidas de dispersión o tendencia central según su naturaleza.

\section{Consideraciones éticas}

El estudio fue autorizado por el Comité de Ética de la Facultad de Medicina de la Universidad Nacional de Colombia.

\section{Resultados}

Durante el periodo comprendido entre el 15 de agosto de 2011 y el 15 de febrero de 2012, hubo 2.007 nacimientos en el Hospital La Victoria, sede Instituto Materno Infantil, entre los cuales se detectaron 29 casos de sífilis congénita; de ellos, en uno hubo muerte fetal $(3,5 \%)$ y los otros 28 cumplieron con los criterios de la definición de caso de sífilis congénita (figura 1), con una razón de un caso por cada 69 recién nacidos y una incidencia de 14,5 por 1.000 nacimientos. De los 28 recién nacidos vivos, 13 tuvieron manifestaciones biológicas y clínicas (46,4\%) y 15 no las presentaron (53,6 \%). Las características generales de los recién nacidos se describen en los cuadros 1 y 2.

Las 29 mujeres gestantes fueron negativas en las pruebas de HIV y de antígeno de superficie para hepatitis B. Veinte de ellas no asistieron a control prenatal $\mathrm{y}$, por lo tanto, no recibieron tratamiento; de las nueve mujeres gestantes que asistieron a control prenatal, no se encontró registro de la

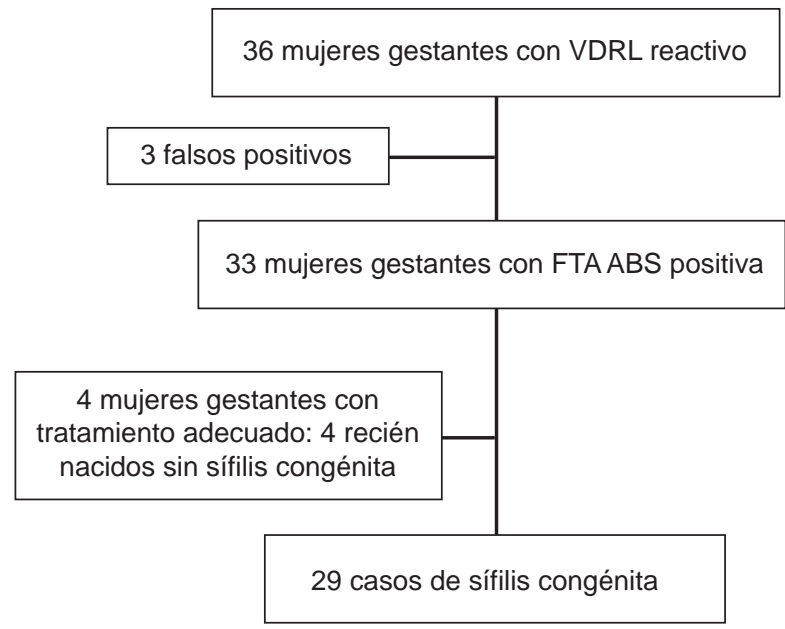

Figura 1. Mujeres gestantes con VDRL reactivo y casos de sífilis congénita
Cuadro 1. Características generales de los recién nacidos vivos diagnosticados con sífilis congénita

\begin{tabular}{llrc}
\hline Variable & Categorías & n & $\%$ \\
\hline Sexo & Masculino & 12 & 42,9 \\
& Femenino & 16 & 57,1 \\
Enfermedad materna asociada* & Sí & 6 & 21,4 \\
& No & 22 & 78,6 \\
Vía del nacimiento & Vaginal & 20 & 71,4 \\
& Abdominal & 8 & 28,6 \\
Tratamiento materno & No & 20 & 71,4 \\
& Inadecuado & 8 & 28,6 \\
Prematuridad & Sí & 4 & 14,3 \\
& No & 24 & 85,7 \\
Peso para la edad de gestación & Bajo & 5 & 17,9 \\
& Adecuado & 23 & 82,1 \\
Compromiso de órganos & Sí & 13 & 46,4 \\
o sistemas & No & 15 & 53,6 \\
\hline
\end{tabular}

* Farmacodependencia: cuatro casos; obesidad: un caso; condilomatosis: un caso

Cuadro 2. Variables antropométricas de los recién nacidos vivos con diagnóstico de sífilis congénita

\begin{tabular}{lcc}
\hline Variables & Promedio & $\begin{array}{c}\text { Desviación } \\
\text { estándar }\end{array}$ \\
\hline Peso $(\mathrm{g})$ & $2.691,86$ & 407,261 \\
Talla $(\mathrm{cm})$ & 47,78 & 2,325 \\
Perímetro cefálico $(\mathrm{cm})$ & 33,26 & 1,868 \\
\hline
\end{tabular}

VDRL para una de ellas, cuyo hijo nació muerto; el diagnóstico de sífilis se hizo en el momento del parto, por lo que la madre no recibió tratamiento. Las otras ocho mujeres gestantes recibieron tratamiento inadecuado porque en tres de ellas este fue tardío (menos de 30 días antes del parto); otra madre recibió el tratamiento, pero tuvo un aumento de los títulos en la prueba de VDRL en el momento del parto comparada con los resultados de la VDRL de diagnóstico; y en cuatro de los casos no hubo disminución de los títulos en la VDRL en el momento del parto comparados con los registrados en la VDRL en el momento del diagnóstico.

Con respecto a los resultados de la VDRL materna, en siete casos no hubo diluciones, en cuatro, una dilución, en cinco, dos diluciones, en otros cinco, cuatro diluciones, en otros cinco se registraron ocho diluciones, en otro, 32 diluciones, y en dos casos, 64 diluciones; en uno de los casos hubo óbito fetal. Todas las mujeres gestantes fueron positivas en la prueba treponémica. No se encontró relación entre los elevados títulos maternos en la VDRL y los síntomas en el recién nacido $(p=0,667)$.

La condición más frecuentemente asociada en las madres fue la farmacodependencia (cuatro casos de $29 ; 13,8 \%$ ), pero la relación con la prematuridad, 
el bajo peso al nacer o el compromiso de órganos y sistemas, no fue estadísticamente significativa $(p=0,1048)$. Hubo un caso con antecedente de sífilis en una gestación previa.

\section{Manifestaciones biológicas y clínicas}

De los 28 recién nacidos, cuatro fueron prematuros $(14,3 \%)$, uno de ellos extremo, y cinco tuvieron peso bajo para la edad de gestación (17,9 \%); en 13 se evidenció compromiso de órganos. Ningún paciente presentó compromiso óseo, ocular o hidropesía fetal; en dos recién nacidos hubo compromiso de la piel y, en otro, compromiso pulmonar. A todos los recién nacidos se les hicieron exámenes de cuadro hemático, parcial de orina, punción lumbar y radiografía de huesos largos, y se encontró alteración hematológica (trombocitopenia) en cuatro (14,3\%), alteración renal (hematuria, proteinuria, cilindruria), en cinco $(17,9 \%)$, y del sistema nervioso central en dos $(7,1 \%)$, uno de ellos con VDRL reactiva en líquido cefalorraquídeo y examen citoquímico normal, y el otro con aumento de proteínas y VDRL no reactiva.

Se registró un aumento de aminotransferasas en siete pacientes de nueve que tenian reporte de función hepática, colestasis en tres de 16 con reporte de bilirrubinas e hipoalbuminemia, y prolongación de los tiempos de coagulación en uno de estos casos. En todos los siete casos de pacientes con compromiso hepático hubo aumento de la aspartato aminotransferasa (AST) y un caso presentó aumento de la alanino aminotransferasa (ALT).

En cuanto a los títulos en la prueba de VDRL en los recién nacidos, la distribución fue la siguiente: en nueve de ellos, la VDRL no fue reactiva; en tres no se registraron diluciones; en cinco se registró una dilución; en otros cinco, dos diluciones; en dos, cuatro diluciones, y en los otros cuatro, ocho diluciones; en un caso, los títulos en la VDRL fueron mayores por una dilución que los de la VDRL en la madre, caso este en el que se registraron síntomas graves de la enfermedad.

Hubo un caso de muerte neonatal $(3,6 \%)$ a los cuatro días del nacimiento; se trataba de un recién nacido prematuro de 24 semanas y $840 \mathrm{~g}$ de peso con compromiso pulmonar y hepático, que tuvo el valor más alto de AST en la serie.

\section{Seguimiento}

Se citaron para seguimiento 27 pacientes (11 sintomáticos y 16 asintomáticos), pues un recién nacido sintomático falleció por compromiso multisistémico y sepsis neonatal durante el tratamiento. Hubo una pérdida de nueve casos (33\%) durante el seguimiento; los otros 18 pacientes (67\%),de los cuales nueve fueron sintomáticos y nueve asintomáticos, se evaluaron a los tres meses y todos cumplieron con los criterios de curación: VDRL negativa o descenso de dos diluciones en el número inicial de títulos. A los seis meses, seis de los pacientes asistieron al control, incluidos dos casos con pruebas de VDRL ligeramente reactivas frente al control de los tres meses; todos registraron resultados negativos en los títulos de la VDRL. De los 11 pacientes sintomáticos vivos, los nueve que fueron llevados a control a los tres meses presentaban una adecuada evolución clínica y paraclínica.

De los tres pacientes con aumento de aminotransferasas y colestasis, uno de ellos, hijo de madre farmacodependiente, fue tomado en protección por el Instituto Colombiano de Bienestar Familiar y no asistió al control; en el control a los tres meses, se encontró que uno de los otros pacientes registraba un nivel de aminotransferasas levemente elevado y en el otro los valores estaban normales.

De los pacientes con diagnóstico de neurosífilis, uno tuvo control de VDRL en líquido cefalorraquídeo a los tres meses y registró un resultado negativo, y en el otro caso, el control se hizo a los siete meses y se observó un valor normal de proteínas y un resultado negativo en la prueba de VDRL.

\section{Discusión}

A nivel mundial hay un aumento de las enfermedades de transmisión sexual; a pesar de que existe una prueba diagnóstica y un tratamiento eficaz para la infección por $T$. pallidum, cada año se presentan 11 millones de infecciones nuevas y cerca de 1,5 millones de mujeres gestantes se infectan (15). Se considera que la mitad de las mujeres gestantes infectadas no recibe tratamiento, lo que ocasiona un incremento de los casos de sífilis congénita $y$, por ende, mortalidad fetal, neonatal, prematuridad, restricción del crecimiento y neonatos con manifestaciones de la infección.

En el 2014, año del presente estudio, se notificaron en Colombia 1.583 casos de sífilis congénita para una incidencia de 2,35 casos por 1.000 nacidos vivos; la mayor incidencia se registró en Chocó, con 12,8, seguido de Guainía, con 5,9, Guaviare, con 5,7, San Andrés, con 4,8, y Valle, con 4; Bogotá presentó una incidencia de 2 por 1.000 nacidos vivos (16). 
Las fallas en el control prenatal son un factor fundamental para la presentación de la sífilis congénita; en ocasiones, las mujeres gestantes no asisten al control, situación que fue evidente en esta cohorte, en la que 20 de 29 (69 \%) mujeres gestantes no asistieron a control prenatal, y el diagnóstico de sífilis solo se hizo en el momento del parto, condición también informada en un estudio en Buenaventura en el que 28 de 92 (30\%) mujeres gestantes no asistieron al control prenatal. Esta situación debe explorarse para conocer cuáles son los motivos por los cuales las mujeres gestantes no asisten al control, de manera que puedan establecerse estrategias para brindar cuidados prenatales a todas ellas.

Otra circunstancia igualmente preocupante es que el control prenatal es inadecuado, pues en ocasiones no se practica la prueba para sífilis a las madres y, en otras, no se les da tratamiento; en el presente estudio, el resultado de la prueba de VDRL en una de las nueve mujeres gestantes que asistió a control prenatal no se encontró, por lo que no fue diagnosticada ni recibió tratamiento, y la gestación terminó en el alumbramiento de un mortinato por sífilis. En el estudio en Buenaventura, 64 mujeres gestantes asistieron, por lo menos, a un control prenatal, a 59 de ellas se les practicó la prueba para sífilis, pero solo cinco recibieron el tratamiento adecuado (17).

Esta realidad podría modificarse con el uso de pruebas treponémicas rápidas durante la consulta prenatal, las cuales permiten la detección del riesgo $y$, en caso de resultar positivas, la administración de una dosis de 2'400.000 unidades de penicilina benzatínica, con el propósito de prevenir la sífilis congénita (15). Asimismo, deben solicitarse las pruebas no treponémicas para establecer si se trata de una sífilis activa y, en ese caso, completar el tratamiento. Hay suficiente evidencia de que esta estrategia sirve para prevenir la mortalidad fetal y neonatal ocasionada por la sífilis en la mujer gestante (18). La actual guía de práctica clínica para sífilis de la mujer gestante y sífilis congénita, incluye la recomendación del uso de pruebas treponémicas rápidas como tamización para sífilis durante la consulta prenatal.

La relación entre el consumo de sustancias psicoactivas, específicamente la cocaína, y la sífilis, se viene mencionando desde hace varias décadas (19), por lo que en la población de mujeres farmacodependientes debería hacerse detección temprana de la gestación y búsqueda activa de infecciones asociadas, como el sida, la sífilis y la tuberculosis. Llama la atención que en una población de mujeres gestantes de alto riesgo de enfermedades de transmisión sexual, como la del presente estudio, no se encontrara ningún caso positivo de HIV; sin embargo, en una serie de casos de nuestra institución en el 2008, de 70 casos de mujeres gestantes con sífilis, dos de ellas (2,85\%) presentaban HIV (12).

La modificación en la definición del tratamiento inadecuado de las mujeres gestantes asumida en nuestra institución constituye una limitación del presente estudio, pero dicha modificación obedece a la alta prevalencia que se presenta en este hospital debido, fundamentalmente, a los factores de riesgo de la población que se atiende: inicio temprano de la actividad sexual, promiscuidad, consumo de alcohol o sustancias psicoactivas, bajo nivel socioeconómico, dificultad en el acceso a los servicios de salud, ausencia o inadecuado control prenatal, y ausencia de tratamiento a la pareja, lo que se suma al fracaso del tratamiento, sobre todo de la sífilis secundaria $(14,19,20)$, cuando los títulos de la VDRL materna en el momento del diagnóstico y en el momento del parto son elevados o cuando no se sabe la duración de la infección, ya que el tratamiento durante la gestación puede modificar la gravedad de la enfermedad sin erradicar las manifestaciones clínicas en el neonato $(21,22)$.

En ocho de los casos de esta cohorte, se consideró que el tratamiento materno había sido inadecuado: en tres casos por haber sido tardío, con el resultado de dos neonatos sin evidencia de compromiso de órganos, y un neonato con compromiso hepático y renal; en un caso, los títulos de la VDRL en el momento del parto ascendieron con respecto a los registrados en el momento del diagnóstico, y el neonato fue prematuro con restricción del crecimiento intrauterino y compromiso hepático; en cuatro casos no hubo disminución de los títulos, y el resultado fueron dos recién nacidos sin evidencia de compromiso de órganos, un prematuro y otro con neurosífilis y compromiso renal, lo que valida la recomendación de que los recién nacidos cuyas madres hayan sido tratadas durante la gestación deben ser evaluados clínica y serológicamente para la detección de una eventual sífilis congénita $(19,20)$.

Los resultados de este estudio difieren de la estimación de que la sífilis de la mujer gestante sin tratamiento o con un tratamiento inadecuado, puede terminar en aborto o en mortinatos en $21 \%$ 
de los casos, en muerte neonatal en $9 \%$ de los casos, en prematuridad o restricción del crecimiento intrauterino en $6 \%$ y en neonatos sintomáticos en $16 \%$ (15), y se asemejan, en cambio, a los encontrados en Buenaventura, ya que la mayoría de los neonatos infectados eran asintomáticos, aunque hay que resaltar que la búsqueda del compromiso de órganos no fue uniforme.

El órgano más frecuente y tempranamente comprometido suele ser el hígado (23). En la presente serie hubo tres casos de compromiso hepático grave: dos pacientes con hepatitis anictérica, y el otro con compromiso sistémico, incluida la restricción del crecimiento, hepato-esplenomegalia, y compromiso renal y del sistema nervioso central; en el seguimiento de este caso se encontró que persistía la elevación de las aminotransferasas indicativa de hepatitis crónica ya conocida y notificada desde hacía varios años (24).

La frecuencia de neurosífilis en los pacientes sintomáticos fue de $20 \%$, cuando en la literatura científica se reporta hasta en $60 \%$ de los casos, aunque hay que recordar que algunos casos de neurosífilis se diagnostican solamente mediante PCR o el test de infección en conejo $(9,25)$.

En cuanto al otro objetivo de este estudio, es decir, la descripción del seguimiento clínico y serológico, son pocos los estudios de seguimiento de los neonatos con sífilis congénita. En la serie de Singh (25) se registraron casos de persistencia de los títulos al final del seguimiento, solo en dos de los cinco se inició el tratamiento al nacer y la mayoría tenía títulos muy altos en el momento del diagnóstico, lo que difiere de la situación de los recién nacidos en este estudio, en quienes el diagnóstico y el tratamiento se hicieron al nacer y los títulos en la VDRL fueron bajos. El seguimiento a los tres meses se hizo en el $67 \%$ de los casos y se encontraron resultados negativos o una disminución de dos diluciones en la prueba de VDRL, en tanto que, en el seguimiento de los tres casos de hepatitis, se encontró la resolución de uno de ellos, hepatitis crónica en otro y el tercer paciente no asistió a los controles de seguimiento. En los dos casos de neurosífilis se cumplieron los criterios de curación: a los tres meses en uno de ellos y a los siete meses en el otro.

El seguimiento es importante, pues pueden presentarse secuelas como anemia, hepatitis crónica, nariz en silla de montar, deficiencia de la hormona de crecimiento, compromiso del primero y octavo pares craneales, hidrocefalia, atrofia cerebral, epilepsia, microcefalia y retardo del neurodesarrollo. Se recomienda, asimismo, que los recién nacidos cuyas madres hayan sido tratadas durante la gestación tengan control, pues se han encontrado secuelas de la infección en el largo plazo (26).

A pesar de que no se ha informado resistencia de T. pallidum a la penicilina, y que las pruebas para el diagnóstico y la penicilina son baratas, la morbilidad ocasionada por la infección transplacentaria es elevada y genera costos considerables para el sistema de salud, situación que puede modificarse con medidas sencillas, económicas e integrales que fomenten la asistencia de las mujeres gestantes al control prenatal, para administrarles el tratamiento en la misma consulta en la que se hace el diagnóstico.

La sífilis congénita es una enfermedad prevenible, y la implementación de los lineamientos de la actual guía de práctica clínica en todos los organismos de salud encargados de la atención de las mujeres gestantes y los recién nacidos permitiría disminuir la frecuencia de la infección y cumplir con uno de los objetivos del milenio, tarea en la que llevamos muchos años de retraso.

\section{Agradecimientos}

Al Hospital La Victoria, sede Instituto Materno Infantil.

\section{Conflicto de intereses}

No hay conflictos de intereses.

\section{Financiación}

División de Investigación de la Universidad Nacional de Colombia.

\section{Referencias}

1. Walker GJA, Walker DG. Congenital syphilis: A continuing but neglected problem. Semin Fetal Neonatal Med. 2007;12: 198-206. http://dx.doi.org/10.1016/j.siny.2007.01.019

2. World Health Organization. Investment case for eliminating mother-to-child transmission of syphilis: Promoting better maternal and child health and stronger health systems. 2012. Fecha de consulta: 23 de abril de 2014. Disponible en: http://apps.who.int/iris/bitstream/10665/75480/1/9789 241504348_eng.pdf.

3. Alonso M. Regional Initiative for the Elimination of Motherto-Child Transmission of HIV and Congenital Syphilis in Latin America and the Caribbean: Regional Monitoring Strategy. PAHO, WHO, Unicef; 2010. Fecha de consulta: 23 de abril de 2014. Disponible en: http://www.unicef.org/lac/ Regional_Monitoring_Strategy.pdf.

4. Alzate-Granados JP, Sánchez-Bello NF, Amaya-Arias AC, Peralta-Pizza F, Eslava-Schmalbach J. Congenital syphilis incidence disparities in Colombia 2005 to 2011: An ecological study. Rev Salud Pública. 2012;14:71-80. 
5. Casas RL, Rodríguez M, Rivas J. Syphilis and pregnancy: Early diagnosis and timely treatment. Rev Colomb Obstet Ginecol. 2009;60:49-56.

6. Herremans T, Kortbeek L, Notermans DW. A review of diagnostic tests for congenital syphilis in newborns. Eur $\mathrm{J}$ Clin Microbiol Infect Dis. 2010;29:495-501. http://dx.doi. org/10.1007/s10096-010-0900-8.

7. Woods CR. Syphilis in children: Congenital and acquired. Semin Pediatr Infect Dis. 2005;16:245-57. http://dx.doi. org/10.1053/j.spid.2005.06.005

8. Wilson CB, Nizet V, Remington JS, Klein JO, Maldonado Y. Infectious diseases of the fetus and newborn. Philadelphia: Elsevier; 2010. p. 7085.

9. Sánchez PJ, Wendel GD, Grimprel E, Goldberg M, Hall $\mathrm{M}$, Arencibia-Mireles $\mathrm{O}$, et al. Evaluation of molecular methodologies and rabbit infectivity testing for the diagnosis of congenital syphilis and neonatal central nervous system invasion by Treponema pallidum. J Infect Dis. 1993;167:14857. http://dx.doi.org/10.1093/infdis/167.1.148

10. Michelow IC, Wendel GD, Norgard MV, Zeray F, Leos NK, Alsaadi R, et al. Central nervous system infection in congenital syphilis. N Engl J Med. 2002;346:1792-8. http:// dx.doi.org/10.1056/NEJMoa012684

11. Rawtron SA, Mehta S, Bromberg K. Evaluation of a Treponema pallidum-specific IgM enzyme immunoassay and Treponema pallidum western blot antibody detection in the diagnosis of maternal and congenital syphilis. Sex Transm Dis. 2004;31:123-6.

12. Cifuentes $\mathbf{Y}$, Ojeda C. Sífilis congénita en el Instituto Materno Infantil-Hospital La Victoria durante el año 2008. Rev Salud Pública. 2013;15;434-45.

13. Saloojee H, Velaphi S, Goga Y, Afadapa N, Steen R, Lincetto $\mathbf{O}$. The prevention and management of congenital syphilis: An overview and recommendations. Bull World Health Organ. 2004;82:424-30.

14. Alexander JM, Sheffield JS, Sánchez PJ, Mayfield J, Wendel GD. Efficacy of treatment for syphilis in pregnancy. Obstet Gynecol. 1999;93:5-8.

15. Instituto Nacional de Salud. Sífilis congénita. Semana epidemiológica número 53 de 2014 (28 de diciembre al 03 de enero de 2015). Fecha de consulta: 10 de abril de 2015. http://www.ins.gov.co/boletin-epidemiologico/Boletn\%20 Epidemiolgico/2014\%20Boletin\%20epidemiologico\%20 semana\%2053.pdf.

16. Cruz AR1, Castrillón MA, Minotta AY, Rubiano LC, Castaño MC, Salazar JC. Gestational and congenital syphilis epidemic in the Colombian Pacific Coast. Sex Transm Dis. 2013; 40:813-8. http://dx.doi.org/10.1097/ OLQ.0000000000000020

17. Jafari Y, Peeling RW, Shivkumar S, Claessens C, Joseph L, Pai NP. Are Treponema pallidum specific rapid and pointof-care tests for syphilis accurate enough for screening in resource limited settings? Evidence from a meta-analysis. PloS One. 2013;8:e54695. http://dx.doi.org/10.1371/journal. pone.0054695.

18. Sison CG, Ostrea EM, Reyes MP, Salari V. The resurgence of congenital syphilis: A cocaine-related problem. J Pediatr. 1997;130:289-92. http://dx.doi.org/10.1016/S00223476(97)70357-3

19. World Health Organization. Investment case for eliminating mother-to-child transmission of syphilis: Promoting better maternal and child health and stronger health systems. 2012. Fecha de consulta: 23 de abril de 2014. Disponible en: http://apps.who.int/iris/bitstream/10665/75480/1/97892 41504348_eng.pdf.

20. Rawstron SA, Bromberg K. Failure of recommended maternal therapy to prevent congenital syphilis. Sex Transm Dis. 1991;18:102-6.

21. Winscott M, Taylor MM, Kenney K. Identifying unreported and undiagnosed cases of congenital syphilis in Arizona using live birth and fetal death registries. Sex Transm Dis. 2010;37:244-7. http://dx.doi.org/10.1097/OLQ. 0b013e3181c37e2a

22. McFarlin BL, Bottoms SF, Dock BS, Isada NB. Epidemic syphilis: Maternal factors associated with congenital infection. Am J Obstet Gynecol. 1994;170:535-40. http:// dx.doi.org/10.1016/S0002-9378(94)70223-3

23. Hollier LM, Harstad TW, Sánchez PJ, Twickler DM, Wendel GD. Fetal syphilis: Clinical and laboratory characteristics. Obstet Gynecol. 2001;97:947-53.

24. Anand NK, Chellani HK, Wadhwa A, Prasanna SB, Mohan M. Congenital syphilitic hepatitis. Indian Pediatr. 1991;28:157-9.

25. Singh AE, Guenette T, Gratrix J, Bergman J, Parker P, Anderson B, et al. Seroreversion of treponemal tests in infants meeting Canadian surveillance criteria for confirmed early congenital syphilis. Pediatr Infect Dis J. 2013;32:199202. http://dx.doi.org/10.1097/INF.0b013e318273599c.

26. Lago EG, Vaccari A, Fiori RM. Clinical features and followup of congenital syphilis. Sex Transm Dis. 2013;40:85-94. http://dx.doi.org/10.1097/OLQ.0b013e31827bd688 